Revue de recherche interdisciplinaire sur le genre et la sexualité

\title{
Eliane Vogel-Polsky : éminente juriste, féministe engagée, fervente européenne
}

\section{Agnès Hubert}

\section{OpenEdition}

\section{Journals}

Édition électronique

URL : https://journals.openedition.org/sextant/407

DOI : $10.4000 /$ sextant.407

ISSN : 2795-8736

\section{Éditeur}

Éditions de l'Université de Bruxelles

\section{Édition imprimée}

Date de publication : 1 décembre 2018

Pagination : 153-160

ISBN : 978-2-8004-1636-6

ISSN : $1370-267 X$

Référence électronique

Agnès Hubert, "Eliane Vogel-Polsky : éminente juriste, féministe engagée, fervente européenne », Sextant [En ligne], 35 | 2018, mis en ligne le 01 novembre 2021, consulté le 08 décembre 2021. URL http://journals.openedition.org/sextant/407 ; DOI : https://doi.org/10.4000/sextant.407

\section{c) (1)(2)}

La revue Sextant est mise à disposition selon les termes de la Licence Creative Commons Attribution Pas d'Utilisation Commerciale - Partage dans les Mêmes Conditions 4.0 International. 


\title{
Eliane Vogel-Polsky : éminente juriste, féministe engagée, fervente européenne
}

\author{
Agnès HuberT
}

Eliane Vogel-Polsky s'est éteinte le 13 novembre 2015, quelques heures avant l'horrible massacre du Bataclan, des rues de Charonne, de la Fontaine-au-Roi, du boulevard Voltaire... La presse n'a donc pas été très attentive à sa disparition. Près d'un mois après son décès, seul le journal Le Monde a dressé un joli portrait de cette « défenseure convaincue de l'Europe sociale qui s'est battue tout au long de sa carrière pour faire progresser le droit des femmes ${ }^{1}{ }^{1}$. La presse belge n'a en revanche pas fait écho de sa disparition.

C'était pourtant une grande dame belge qui a fait faire un pas de géant à l'égalité en Belgique et en Europe. Le $1^{\mathrm{er}}$ avril 1976, dans son jugement sur le procès Defrenne II, la Cour européenne de justice reconnaissait que l'article 119 du traité de Rome pouvait être invoqué par les cours nationales et que l'égalité entre les hommes et les femmes était un principe fondateur du droit européen. C'est un progrès fondamental ; l'histoire retiendra que nous le devons à Eliane Vogel-Polsky.

A l'aide de nos conversations, de ses écrits et de la biographie réalisée par les historiennes Eliane Gubin et Catherine Jacques ${ }^{2}$, je retrace ici le parcours singulier d'Eliane, qui a conduit au jugement décisif du $1^{\text {er }}$ avril 1976. Puis, dans un second temps, je présente la suite de son combat pour faire valoir les droits des femmes, ses interrogations, ses intuitions et ses projets pour une Europe plus démocratique et plus sociale.

${ }^{1}$ J.-P. Stroobants, « Eliane Vogel-Polsky, juriste et militante féministe, est morte à 90 ans », Le Monde, 9 décembre 2015.

${ }^{2}$ E. Gubin, avec la collaboration de C. JACQues, Eliane Vogel-Polsky: Une femme de conviction, Bruxelles, Institut pour l'égalité des femmes et des hommes, 2007. 


\section{Le droit européen pour combattre les injustices}

Eliane naquit à Gand le 5 juillet 1926. Dès son berceau, elle est dotée d'une formidable volonté d'arriver à ses fins. Ses parents qui avaient, après la première guerre mondiale, remué des montagnes pour se retrouver ensemble en Belgique lui ont sans nul doute transmis la conviction qu'à cœur vaillant il n'est rien d'impossible. Son père, russe d'origine, venu en Belgique pour faire ses études à la veille de la déclaration de guerre, s'était engagé comme volontaire dans le corps belge des autoscanons-mitrailleuses, envoyé en 1915 sur le front russe. Par une série de hasards, il est arrivé à Kharbin, enclave russe de Mandchourie où il rencontre Nine, sa future femme, chirurgienne dentiste. Leur mariage aura lieu à Gand en 1920 : la société belgo-mandchoue d'exportation de... champagne que Jean Polsky avait créée avec un héritier des champagnes Mercier aura permis de faire venir Nine en Belgique... Rien ne leur avait résisté.

Eliane a, quant à elle, fait l'expérience de l'injustice dès son adolescence. En 1941, à l'âge de 15 ans, elle est sommée de quitter le lycée pour jeunes filles Emile Jacqmain suite à l'ordonnance qui interdit aux enfants de parents juifs de fréquenter des écoles non juives. Elle a poursuivi ses études sous une fausse identité dans un collège de bénédictines à Liège. Cela a-t-il fait partie de ses motivations quant à sa future profession d'avocate ? Bien vite en effet, le droit est devenu une passion pour Eliane : elle décroche son diplôme de docteure en droit à l'Université libre de Bruxelles en 1950. Elle aime le verbe et les joutes oratoires et n'a pas peur de braver un milieu d'avocats et de magistrats qui reste majoritairement hostile à l'ébranlement de la famille et à la décadence de la moralité que représente pour eux l'accès des femmes aux professions juridiques.

Le «féminisme » d'Eliane s'est nourri de la lecture de Simone de Beauvoir, de la misogynie des tribunaux mais aussi du contact avec un groupe de femmes syndicalistes à l'automne 1965. Trois d'entre elles travaillent à la fabrique d'armes d'Herstal. Dans un entretien publié en 2003, dans la revue Raisons politiques, sous le titre «Agir pour les droits des femmes $»^{3}$, elle raconte comment, appelée par la FGTB à animer un séminaire sur le thème de l'Europe et l'égalité des salaires, elle explique alors que la seule norme de droit permettant d'imposer l'égalité des salaires entre hommes et femmes se trouve dans le traité de Rome. Mais l'applicabilité directe de l'article 119, qu'elle s'efforce de faire valoir auprès de ses collègues du Barreau, n'est pas formellement reconnue. Il faut des combats, leur confie-t-elle, pour porter devant la Cour de justice des Communautés européennes la question de l'interprétation de l'article 119. «Il faut trouver un cas car tu ne convaincras personne sans cela », lui avait conseillé Léon-Eli Troclet, son professeur, son mentor, docteur en droit, sénateur, ministre et parlementaire européen dès 1961 qui lui avait transmis le virus européen et le virus social.

L'article 119 du traité de Rome stipule que " chaque Etat membre assure, au cours de la première étape, et maintient par la suite, l'application du principe d'égalité des rémunérations entre les travailleurs masculins et les travailleurs féminins pour

${ }^{3}$ E. Vogel-Polsky, « Agir pour les droits des femmes », Raisons politiques, 10/2, 2003, p. 139-149. 
un même travail ». Or, les « femmes machines » de la Fabrique nationale d'armes de guerre située à Herstal recevaient un salaire inférieur au plus jeune des commis de sexe masculin. Près de dix ans après la naissance de la Communauté économique européenne, ce principe d'égalité de salaires demeurait donc lettre morte. L'article 119 était considéré comme une déclaration d'intention de nature programmatique et non comme une norme devant être transposée dans le droit interne des Etats membres de la Communauté.

En fait, le passage à la deuxième étape de l'intégration en 1961 s'est fait en dépit du fait qu'aucune mesure n'avait été prise par les gouvernements pour assurer le principe d'égalité de rémunération. Manque de données, résistance des décideurs, désintérêt des syndicats, finalement les Etats membres, en pleine contravention à l'engagement qu'ils avaient pris dans le cadre du traité, ont convenu de nommer un « comité article 119 » et d'en passer à la seconde étape. Eliane Vogel-Polsky avait été particulièrement révoltée par le fait que, sur ce seul article, les Etats membres avaient trouvé acceptable de déroger à leurs obligations. Confrontée à la situation déplorable des ouvrières de Herstal dont les conditions de travail étaient particulièrement dures, cette désinvolture était à ses yeux criminelle.

La grève des ouvrières de la Fabrique nationale d'armes de Herstal dura deux mois et demi au printemps 1966 ; lors des manifestations de l'époque, une banderole demandait l'application de l'article 119. Les syndicalistes qui menaient la grève avaient été sensibles à l'argumentation de la jeune avocate qui voyait dans le droit européen un moyen de faire appliquer un principe déjà reconnu par la Société des Nations au lendemain de la première guerre mondiale.

Les ouvrières de Herstal n'ont obtenu qu'une augmentation symbolique de leurs salaires, encore très inférieurs à ceux de leurs collègues masculins. Toute ouvrière qui aurait intenté un procès aurait risqué de perdre son emploi. Les dirigeants syndicaux n'étaient par ailleurs guère disposés à soutenir une action qui aurait menacé le cadre de leur convention collective. Déçue par l'issue de la grève, Eliane Vogel-Polsky n'a eu de cesse à partir de là de chercher un cas à référer à la Cour de justice européenne. Si le droit ne pouvait être obtenu par la négociation ou la grève, il le serait par les tribunaux.

Ce n'est qu'en 1976, après plusieurs essais infructueux, que la jeune avocate au Barreau de Bruxelles, professeure de la Faculté de droit et de l'Institut d'études européennes, a réussi à faire reconnaître par le droit l'application de l'article 119, dans l'affaire de l'hôtesse de l'air Gabrielle Defrenne. Cette affaire avait été précédée de plusieurs essais infructueux, parmi lesquels l'affaire Mertens : dans cette affaire, Eliane Vogel-Polsky était parvenue à faire reconnaître l'injustice du système d'allocations de chômage, fixées à $60 \%$ du salaire pour les hommes et à $40 \%$ seulement pour les travailleuses. Ce procès, gagné par la jeune plaignante de 19 ans, lui avait valu le qualificatif de « femme la plus chère de Belgique ! ». Le ministre du Travail, pourtant ancien syndicaliste, n'avait pas apprécié le supplément de dépense publique au bénéfice des femmes créé par cette jurisprudence ! Eliane Vogel-Polsky n'a toutefois pas atteint son objectif principal lors de ce procès : les allocations de chômage n'étant pas considérées comme faisant partie de la rémunération, la question n'avait pu été référée à la Cour de justice des Communautés européennes. 
Il faudra attendre le $1^{\text {er }}$ avril 1976 pour que l'arrêt Defrenne II c. Sabena couronne l'aboutissement de ce combat de dix ans, créant une jurisprudence qui ouvrira la voie à d'importants développements de la politique européenne d'égalité. La Cour donnera raison à l'hôtesse de l'air de la compagnie Sabena, "remerciée " à l'âge de quarante ans sans indemnité ni reclassement, à la différence des agents de bord masculins qui occupaient un emploi comparable.

L'arrêt Defrenne II a eu un retentissement énorme. La Cour avait mis en évidence la double finalité poursuivie par l'article 119 : une finalité économique, visant à établir une égalité de condition de concurrence entre entreprises des Etats membres, et une finalité sociale, la Communauté devant assurer par une action commune le progrès social et poursuivre l'amélioration constante des conditions de vie et d'emploi des peuples européens. Selon l'attendu 12 de l'arrêt, « de cette double finalité, économique et sociale, il résulte que le principe d'égalité de rémunération des hommes et des femmes fait partie des fondements de la Communauté ». Sur la base de cet arrêt, qui précise et élargit la portée de l'article 119, une politique d'égalité européenne a pu être déployée, non seulement dans le domaine de l'emploi en reconnaissant, au-delà de l'égalité de salaire, l'égalité de traitement, mais aussi dans les domaines social et politique.

De traité en traité, les Etats membres ont renforcé et élargi le «principe fondateur de l'égalité des femmes et des hommes » pour se donner les moyens de traduire l'égalité formelle en égalité réelle. Dans le traité d'Amsterdam de 1997, sous l'effet conjugué d'un mouvement des femmes renforcé par la conférence onusienne de Beijing et de la revendication de parité à laquelle Eliane Vogel-Polsky avait été aussi partie prenante, la garantie de l'égalité entre les hommes et les femmes est reconnue et sa réalisation devient une mission de la Communauté dans les domaines de sa compétence et plus seulement dans le domaine de l'emploi. Le principe d'actions positives est également reconnu dans certaines conditions « en faveur du sexe sous-représenté ». En 2007, l'intégration dans le traité de Lisbonne de la charte des droits fondamentaux a encore renforcé le droit à l'égalité.

\section{Actions positives, démocratie paritaire : la lutte continue pour une Europe démocratique et sociale}

Après l'arrêt Defrenne, Eliane Vogel-Polsky s'est attachée à chercher les moyens juridiques pour transformer l'égalité de jure en égalité réelle. Pour ce faire, elle a exploré le potentiel des actions positives. Nous sommes au début des années 1980. La situation des ouvrières d'Herstal ou des hôtesses de l'air de la Sabena la persuade que l'égalité ne pourra devenir effective sans l'aide de nouveaux outils, pour permettre aux femmes de rattraper leur retard sur les hommes dans la sphère professionnelle. C'est d'autant plus important que, sous le double effet de l'allongement de la durée des études et du développement du secteur des services, les femmes entrent toujours plus nombreuses sur le marché du travail et que l'égalité de rémunération et de traitement est loin d'être acquise.

Le concept d'actions positives est la traduction européenne des « affirmative actions » développées aux Etats-Unis pour donner un coup d'accélérateur à la lutte contre les discriminations raciales. Cela consiste à donner un avantage temporaire aux 
personnes discriminées. La Commission européenne, préoccupée par la faiblesse des résultats obtenus en matière d'égalité dans les Etats membres, confie en 1982 à Eliane Vogel-Polsky la rédaction du premier rapport européen sur le sujet. Celui-ci analyse les stratégies développées aux Etats-Unis et dans les pays scandinaves, établit des comparaisons entre Etats membres et recommande à la Commission de développer des instruments juridiques contraignants pour " corriger les inégalités du passé » en donnant temporairement aux femmes un avantage qui leur permet de rattraper leur retard sur les hommes dans l'emploi... Ce rapport est une déception pour Eliane Vogel-Polsky : la Commission n'a proposé qu'une recommandation (instrument non contraignant) et non une directive. Toutefois, le débat est lancé, un débat controversé, qui ne disparaîtra plus.

Faut-il accorder des avantages spécifiques aux femmes, y compris par l'établissement de quotas ? Les opposants aux actions positives, au nom de la nondiscrimination, considèrent que celles-ci doivent être limitées à des mesures de type formation ou garde d'enfants pour aider les femmes à assumer leur emploi. De l'autre côté, les féministes, elles, s'insurgent contre des mesures qui érigent le modèle masculin comme objectif à atteindre. Aujourd'hui encore, la question des actions positives sous forme de quotas reste contestée : c'est une raison qui explique que l'adoption de la proposition de directive sur la participation accrue des femmes dans les conseils d'administration (women on boards) est toujours bloquée par les ministres européens.

Il n'en reste pas moins que cette graine plantée par Eliane dans le champ de la politique européenne d'égalité a été un pilier essentiel alors que l'introduction du gender mainstreaming (intégration de l'égalité dans toutes les politiques) menaçait de diluer les politiques d'égalité. La Commission européenne a en effet alors réaffirmé que la réalisation de l'égalité s'appuyait sur une « double approche » comportant des actions positives et le mainstreaming de l'égalité. Ces controverses ont aussi préparé le terrain pour que la possibilité de prendre « des mesures en faveur du sexe sous-représenté » afin d'éliminer les inégalités professionnelles, figure dans le traité (article 153).

A l'écoute des arguments des uns et des autres, Eliane a saisi, dès qu'elle s'est présentée, l'occasion de transformer en enjeu politique la controverse sur les quotas. En tant que représentante de la Belgique au sein du groupe européen d'expertes sur les « femmes dans la prise de décision », elle a défendu en droit l'idée de « démocratie paritaire » en plaçant ce concept au cœur de la construction d'une " citoyenneté européenne » inscrite pour la première fois dans le traité d'Amsterdam. La parité, qui dépasse la notion de référent masculin contenue dans l'idée d'action positive (rattrapage par rapport à la situation de l'homme) apparaît comme la clé pour dépasser l'opposition des défenseurs de l'anti-discrimination et des féministes. Les raisons pour lesquelles il faut réaliser un véritable équilibre hommes-femmes dans la politique comme sur le marché du travail, voire dans la famille, ne sont pas corporatistes, c'est une nécessité démocratique.

Au sein du réseau européen «Femmes et prise de décision », la juriste Eliane VogelPolsky, la jeune avocate devenue sénatrice Sabine de Béthune et Françoise Gaspard, ancienne députée européenne, femme politique française et historienne, ont joué un 
rôle de premier plan pour justifier et mener le combat pour la démocratie paritaire. En 1992, alors que l'on fête le bicentenaire de l'instauration de la République française, il apparaît avec évidence que le citoyen universel, neutre et asexué a été érigé par les Conventionnels sur l'exclusion politique des femmes et leur incapacité civile. Il est impossible dans ce cas d'inscrire l'égalité entre les femmes et les hommes dans nos Constitutions et dans les traités européens sans revoir la notion même d'égalité, qui vient du siècle des Lumières. Eliane Vogel-Polsky et le réseau ont défendu le principe qu'aucune démocratie réelle n'est possible si la question de l'égalité entre les femmes et les hommes n'est pas posée comme un préalable politique, relevant des principes constitutifs du régime, exactement comme le suffrage universel ou la séparation des pouvoirs. Cette notion rompt complètement avec l'ancienne idée d'égalité dans la mesure où le masculin n'est plus le référent et où les deux sexes bénéficient désormais d'une égale légitimité.

La notion de démocratie paritaire et le concept de parité de représentation dans les instances de pouvoir se sont diffusés comme une traînée de poudre à un moment où la sous-représentation des femmes, en politique en particulier, apparaissait comme choquante. Au début des années 1990, l'Europe communautaire compte en moyenne $11 \%$ de femmes dans les parlements nationaux, 19\% au Parlement européen. L'action du réseau (y compris la campagne menée par Eliane qui s'est portée candidate... « ne prenons pas l'excuse du manque de femmes », disait-elle, «pour être cohérentes, prouvons qu'elles sont là ») a porté ce pourcentage à $27 \%$ après les élections européennes de $1994^{4}$.

La démocratie paritaire, c'est à la fois une question de justice parce que l'égalité $\mathrm{H} / \mathrm{F}$ est un droit fondamental et que les femmes représentent plus de la moitié de la population. C'est aussi une question d'efficacité, parce que les femmes représentent la moitié des talents et qualifications potentielles de l'humanité et que leur absence des instances de pouvoir constitue une perte pour la société dans son ensemble. C'est enfin le stimulant d'un renouveau parce qu'une participation équilibrée est susceptible d'engendrer des idées, des valeurs et des comportements différents allant dans le sens d'un monde plus juste et équilibré pour les femmes et pour les hommes.

La conception de la démocratie d'Eliane Vogel-Polsky est indissociable de la construction européenne et la construction européenne est indissociable de ce qu'elle a engendré par hasard : l'égalité des femmes et des hommes. Ce mélange s'est appelé « la citoyenneté européenne et les femmes », une étude qu'elle a remis à la Commission européenne en 1994 « car le concept de citoyenneté européenne apparu en 1984 au conseil de Fontainebleau puis au conseil européen de Dublin en 1990 sera le ferment novateur de la future constitution européenne. L'Europe doit saisir cette occasion de redéfinir les exigences et le contenu d'une citoyenneté active et responsable, qui arrime la question de la représentation équilibrée des hommes et des femmes $\gg{ }^{5}$. Cette

${ }^{4}$ Sur l'action du réseau « Femmes et prise de décision », voir A. HuBERT, « Du sommet d'Athènes à la révision de la constitution, les dogmes de la république à l'épreuve de la démocratie paritaire en Europe », in B. Perreau et J. W. Scott (dir.), Les défis de la République, Genre, territoires, citoyenneté, Paris, Presses de Sciences Po, 2017, p. 113-135.

5 E. Vogel-Polsky, Les femmes et la citoyenneté européenne. Recherche menée pour l'Unité pour l'égalité des chances DGV de la Commission des Communautés Européennes, 
étude dresse un véritable cahier des charges sur les mesures à prendre pour établir cette citoyenneté. Quelques années plus tard, dans la mouvance de cette idée, elle sera la seule à souligner combien la nécessité de formuler et d'expérimenter de nouvelles formes de gouvernance en Europe est l'occasion de bénéficier de la formidable énergie et des talents des femmes en mettant en œuvre de nouvelles formes de participation.

Consultante auprès du Conseil des communes et régions d'Europe, Eliane VogelPolsky a aussi été l'auteure de la «Charte de l'égalité des femmes et des hommes dans la ville », un texte d'une remarquable précision sur les mesures à prendre pour réaliser une ville égalitaire. Cette charte est aujourd'hui ratifiée par plus de 1500 collectivités territoriales européennes et, pour en réaliser le suivi, le Conseil des communes et régions d'Europe vient de mettre au point une série d'indicateurs ${ }^{6}$.

\section{Conclusion}

Le parcours d'Eliane Vogel-Polsky est riche d'enseignements à plusieurs égards : enfant d'un siècle de grands changements, née dans une famille russe en Belgique, juive ayant subi la persécution nazie, reconnaissante envers les carmélites qui l'ont élevée puis rejoignant une université laïque et encore très masculine, son parcours initial la prédisposait à être sensible aux injustices. Ses rencontres, dont celle de Léon-Eli Troclet, forgent son engagement de militante socialiste et européenne, mais ce n'est qu'au contact de la réalité que vivent les « femmes machines » de la Fabrique nationale d'armes de Herstal que le parcours d'Eliane est devenu singulier et remarquable. A partir de là, c'est avec un engagement qui ne l'a jamais quittée qu'elle a entamé son combat pour les droits des femmes, comme avocate, comme professeure, comme experte internationalement reconnue.

Eliane fait partie de ces intellectuelles qui ne se satisfont jamais d'une avancée, si importante fût-elle. Ses victoires, pourtant nombreuses à partir de l'arrêt Defrenne, ne sont jamais complètes : elles la poussent à plus d'exigence et à poursuivre sa lutte. C'est sur ce mode que se sont enchaînés ses combats pour les actions positives, pour la démocratie paritaire, pour la citoyenneté européenne et pour une gouvernance démocratique de l'Europe.

Le destin singulier de cette grande juriste, passionnément féministe et européenne fervente, nous laisse en héritage un modèle mais aussi une méthode et des outils. Utilisant le droit comme instrument au service de la justice sociale, et l'Europe comme terrain nouveau de réalisation de la démocratie, elle a poursuivi avec succès une intuition initiale. La prise en considération égale des femmes et des hommes est le fondement d'une société harmonieuse que l'Europe unie a les moyens de construire. Elle en a forgé les modes de faire : en appliquant le droit communautaire, en développant des outils, telles les actions positives, mais aussi la démocratie paritaire fondée sur une citoyenneté européenne active et responsable et des institutions participatives et paritaires. Sa vision d'une Europe plus juste pourrait nous paraître bien lointaine, alors que le populisme et l'extrême droite ont gagné du terrain et que

Etude $\mathrm{n}^{\circ}$ 93.0228, Bruxelles, Commission européenne, 1994.

${ }^{6} \mathrm{http}: / / \mathrm{www}$.afccre.org/fr/dossiers-thematiques/egalit\%C3\%A9-femmes-hommes\#. WSG_4LzyjfY. 
les domaines de solidarité européenne ont été affaiblis sous l'effet de la crise. En raison aussi de la régression en matière de droits des femmes que nous constatons, nous avons d'autant plus besoin aujourd'hui de reprendre l'héritage d'Eliane VogelPolsky et de poursuivre son utopie d'une Europe plus juste. Aujourd'hui plus que jamais, son message doit être transmis aux jeunes générations. Une Europe juste, sociale est possible, à condition qu'elle soit paritaire. 